\title{
Influência da distância na exatidão da cubagem não destrutiva de Qualea sp. no bioma Amazônia
}

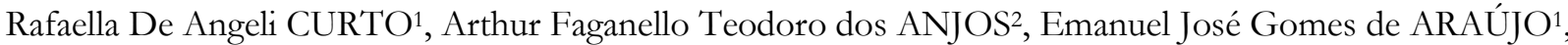 \\ Charlote WINK ${ }^{2}$, Sintia Valerio KOHLER ${ }^{3}$
}

\author{
${ }^{1}$ Universidade Federal Rural do Rio de Janeiro, Seropédica, RJ, Brasil. (Orcid: *; 0000-0002-2301-1031) \\ ${ }_{2}^{2}$ Instituto de Ciências Agrárias e Ambientais, Universidade Federal de Mato Grosso, Sinop, MT, Brasil. \\ (Orcid: 0000-0002-9620-0781; 0000-0001-7066-7028) \\ ${ }^{3}$ Universidade Federal Rural do Amazonas, Parauapebas, PA, Brasil. (Orcid: 0000-0002-8532-771X) \\ *E-mail: rafaellacurto@yahoo.com.br (Orcid: 0000-0001-5509-4655)
}

\begin{abstract}
Recebido em 18/07/2020; Aceito em 02/10/2020; Publicado em 27/10/2020.
RESUMO: Objetivou-se avaliar o efeito da distância na exatidão do dendrômetro digital Criterion RD $1000^{\circledR}$, na estimativa do diâmetro e volume de Qualea sp. no bioma Amazônia. Foram selecionadas 30 árvores e cubadas pelo método de Smalian até a altura comercial com escalada e com Criterion RD $1000^{\circledR}$, nas distâncias entre observador e a árvore de $11 \mathrm{~m}, 13 \mathrm{~m}, 15 \mathrm{~m}$ e à maior distância que o observador conseguiu instalar o equipamento em relação a árvore. Para avaliar o diâmetro ao longo do fuste, o volume comercial e por segmento, realizou-se o teste $t$ pareado, com $95 \%$ de probabilidade, a análise de resíduos e as estatísticas complementares. Não houve diferença significativa entre os valores médios das variáveis analisadas, obtidos com a escalada e os estimados pelo Criterion. Houve menor amplitude dos resíduos nas seções inferiores do fuste com tendência em subestimar os menores diâmetros. As estatísticas complementares demonstraram maiores erros quando o equipamento estava mais próximo da árvore e menores quando estava a $15 \mathrm{~m}$, correspondendo a uma distância superior à média da altura comercial das árvores. O equipamento permitiu estimar com exatidão diâmetros e volumes de árvores em pé, viabilizando a cubagem não destrutiva de Qualea sp. no bioma Amazônia.
\end{abstract}

Palavras-chave: mensuração; volumetria; Criterion RD $1000^{\circledR}$.

\section{Influence of distance on the accuracy of the non-destructive of Qualea sp. in the Amazon biome}

\begin{abstract}
The objective was to evaluate the effect of distance on the accuracy of the digital dendrometer Criterion RD $1000 \AA$, on the estimate of the diameter and volume of Qualea sp. in the Amazon biome. Thirty trees were selected and scaled by the Smalian method to commercial height with climbing and with a Criterion $\mathrm{RD} 1000 \AA$, in the distances between the observer and the $11 \mathrm{~m}, 13 \mathrm{~m}, 15 \mathrm{~m}$ and the longest distance that the observer managed to install the equipment in relation to the tree. To analyze diameter along the stem, commercial volume and volume per segment, the $t$ test for dependent samples was performed, with a $95 \%$ probability, as well as residual analysis and complementary statistics. There was no significant difference between the mean values of the variables analyzed, obtained with the climb and estimated with a Criterion. There was a smaller amplitude of residues in the lower sections of the stem with tendency to underestimate smaller diameters. The complementary statistics showed greater errors with the equipment closest to the tree and smaller when it was $15 \mathrm{~m}$ away, corresponding to a distance greater than the average commercial height of the trees. The equipment made it possible to accurately estimate the diameters and volumes of standing trees, enabling the non-destructive scaling of Qualea sp. in the Amazon biome forest.
\end{abstract}

Keywords: measurement; volumetry; Criterion RD $1000^{\circledR}$.

\section{INTRODUÇÃO}

A quantificação do estoque de madeira na floresta é primordial e de interesse de qualquer administrador florestal, independente de imposições da legislação. Assim, ao conhecer o empreendimento sob manejo, obtém-se subsídios adequados para tomada de decisões em relação ao seu uso. $\mathrm{O}$ inventário florestal é uma das estratégias adotadas para o levantamento de matéria-prima estocada em uma área, permitindo quantificar a floresta. Nele, usam-se técnicas indiretas para estimar o volume das árvores, como o fator de forma, equações de volume e funções de afilamento (taper), sendo que, para o desenvolvimento dessas técnicas, é necessário o conhecimento do volume real das árvores (SOUZA et al., 2017; NICOLETTI et al., 2015a). Para encontrar o fator de forma, ajustar equações de volume ou funções de afilamento em florestas plantadas ou nativas, são comumente empregadas metodologias destrutivas, onde deve-se derrubar as árvores para realizar medições de diâmetros e alturas, obtendo-se o volume total e/ou comercial, a partir da soma dos volumes das seções medidas ao longo do fuste (BONAZZA et al., 2015).

Todavia, em casos onde não é possível a derrubada, o avanço tecnológico permitiu o desenvolvimento de equipamentos óticos, capazes de realizar as medições de 
diâmetros e alturas a uma dada distância, não necessitando a derrubada de árvores (NICOLETTI et al., 2012; SANQUETTA et al., 2017). Entre estes equipamentos, destaca-se o Criterion RD $1000^{\circledR}$, que foi desenvolvido baseado em princípios trigonométricos. A exatidão das medidas obtidas por este equipamento pode variar de acordo com a altura da árvore e a distância do operador em relação a mesma (SILVA et al., 2017), visto que em situações específicas o mensurador pode encontrar dificuldades para manuseio ou o próprio equipamento pode apresentar limitações operacionais. Além disso, em florestas nativas existem muitas outras variáveis que podem interferir na leitura do equipamento, dificultando a realização das medidas.

São escassos os trabalhos que avaliam o desempenho do Criterion na avaliação de florestas tropicais, como a Amazônia, a qual apresenta formações bem distintas floristicamente, devido aos variados fatores ambientais que promovem diversas associações entre os componentes bióticos de cada ecossistema (GAMA et al., 2003). Geralmente, nestas florestas, a densidade do sub-bosque tende a dificultar as mensurações, quando comparado aos plantios homogêneos, em que já existem trabalhos comprovando a eficácia do Criterion em distâncias semelhantes à altura das árvores (RODRIGUEZ et al., 2014; BONAZZA et al., 2015; CORTE et al., 2016).

Em Planos de Manejo Florestal Sustentável (PMFS) no bioma Amazônia, o ajuste de equações de volume é um procedimento obrigatório a partir do segundo Plano Operacional Anual (POA), para a estimativa do volume de árvores em pé nas Unidades de Produção Anual (UPA) (BRASIL, 2009). No entanto, em florestas nativas, onde são feitos os PMFS com fins madeireiros, existem imposições normativas que proíbem, entre outros, o abate de árvores com diâmetro inferior a $50 \mathrm{~cm}$ (BRASIL, 2009). Assim, as árvores de menores dimensões podem ser mensuradas pela cubagem não destrutiva com o emprego do Criterion RD $1000 \AA$. Estas árvores são importantes na prognose volumétrica com vistas a subsidiar a redução do ciclo de corte (Resolução CONAMA no 406/2009 e Instrução Normativa $\mathrm{n}^{\mathrm{o}}$ 5/2006 do Ministério do Meio Ambiente), bem como permitir estimativas mais precisas de estoque de carbono na compensação ambiental.

Este estudo foi desenvolvido sob a hipótese de que o aumento da distância exerce efeito positivo no uso do dendrômetro digital Criterion RD $1000^{\circledR}$ para a mensuração acurada de variáveis dendrométricas, sem a necessidade da derrubada das árvores. Dessa forma, o presente trabalho teve como objetivo avaliar o efeito da distância na exatidão do dendrômetro digital Criterion RD $1000^{\circledR}$ na estimativa do diâmetro e volume de árvores no bioma Amazônia.

\section{MATERIAL E MÉTODOS}

\subsection{Caracterização da área}

O estudo foi realizado em um Plano de Exploração Florestal (PEF) com área de 118,57 hectares, (11³6'53,35"S e $\left.55^{\circ} 23^{\prime} 9,02^{\prime \prime O}\right)$, localizado no município de Sinop-MT, a uma altitude média de $375 \mathrm{~m}$. A composição florística e estrutura regional é de transição Cerrado-Floresta Amazônica (ARAÚJO et al., 2009). A área foi explorada em regime de Manejo Florestal Sustentável em 1997, sendo retirados 38,4 $\mathrm{m}^{3}$ de madeira por hectare.

O clima segundo Köppen para a região é do tipo Aw, com estação chuvosa (entre outubro e abril) e seca (de maio a setembro) bem definidas, com precipitação total anual entre 1.300 e $2.000 \mathrm{~mm}$ e temperatura média de 23,5 a $25,5^{\circ} \mathrm{C}$ (ALVARES et al., 2013; MOTA et al., 2013).

\subsection{Coleta de dados}

Foram selecionadas aleatoriamente 30 árvores do gênero Qualea sp. (Cambará) com diâmetros a 1,30 m do solo (DAP) variando de $33 \mathrm{~cm}$ a $78,3 \mathrm{~cm}$ (média de $55,7 \mathrm{~cm}$ e desvio padrão de $12,6 \mathrm{~cm}$ ). Com base na média e no desvio padrão da altura comercial das árvores estimadas visualmente durante o inventário prévio, necessário para aprovação do PEF, foram definidas as distâncias de $11 \mathrm{~m}, 13 \mathrm{~m}, 15 \mathrm{~m} \mathrm{e}$ uma distância máxima em que era possível a visualização da árvore a ser cubada com o dendrômetro digital Criterion RD $1000^{\circledR}$ (Laser Technology Inc.), o qual foi acoplado a um tripé fotográfico profissional.

Para todas as distâncias definidas, as medições com o Criterion foram sempre realizadas na mesma face do fuste da árvore, em que foi realizada a escalada, para isso, a face selecionada foi marcada com tinta spray. Os diâmetros na base (0,1 m do solo) e no DAP foram mensurados com suta. Na sequência, a cubagem foi realizada com o Criterion, a partir de $2 \mathrm{~m}$ de altura e posteriormente a cada $2 \mathrm{~m}$ até a altura comercial do fuste, estabelecida abaixo da primeira bifurcação ou primeiro galho vivo. Após a mensuração com o dendrômetro, procedeu-se à cubagem pela escalada, que consistiu em fixar a trena da base até a altura comercial da árvore com posterior mensuração dos diâmetros utilizando a suta, nas mesmas alturas consideradas na cubagem com o Criterion.

Em ambos os métodos de mensuração (Criterion e escalada), o volume das seções ao longo do fuste foi determinado adotando-se o método de Smalian. O volume comercial de cada fuste foi obtido pela soma dos volumes das seções contidas até a altura comercial da árvore. Para o cálculo do volume, o fuste de cada árvore foi estratificado em dois segmentos, sendo o inferior correspondente as seções entre 2,0 e 8,0 $\mathrm{m}$ de altura e o superior, correspondente às seções a partir dos $8,0 \mathrm{~m}$ de altura até a altura comercial. Utilizou-se esta estratificação para analisar o desempenho do equipamento para medições em diferentes posições do fuste, visto que, para mensuração dos diâmetros no segmento superior o equipamento deve ficar posicionado a uma maior inclinação, podendo gerar erros diferentes do segmento inferior.

\subsection{Análise dos dados}

As variáveis analisadas foram diâmetro ao longo do fuste, volume comercial e volume comercial por segmentos do fuste. Inicialmente realizou-se a análise da homogeneidade das variâncias pelo teste de Bartlett, a 95\% de probabilidade. Constatada homogeneidade, os valores reais (escalada) e estimados (dendrômetro) das variáveis dendrométricas nas diferentes distâncias de mensuração foram comparados entre si pelo teste $t$ pareado, com $95 \%$ de probabilidade.

Além disso, realizou-se a análise gráfica de resíduos para os diâmetros, visando verificar as tendências das estimativas com o Criterion ao longo do fuste. Posteriormente, visando validar as estimativas obtidas com o Criterion, fez-se o ajuste de uma regressão linear simples $\left(y=\beta_{0}+\beta_{1} x+\right.$ ei) para $o$ diâmetro e o volume em todas as variações de distância, sendo que a variável dependente correspondeu aos valores estimados pelo Criterion e a variável independente 
correspondeu aos respectivos valores observados na escalada.

Os coeficientes da regressão foram testados utilizando o teste $\mathrm{t}$, a $95 \%$ de probabilidade considerando a hipótese $\mathrm{H}_{0}$ : $\beta_{0}=0$ e $H_{0}: \beta_{1}=1$. Quando a hipótese de nulidade não é rejeitada, verifica-se que os valores da variável dependente são semelhantes aos valores da variável independente, pois esta relação é retratada como uma linha de regressão com inclinação de $45^{\circ}\left(\beta_{1}=1\right)$ que passa pela origem $\left(\beta_{0}=0\right)$. Com isso foi possível observar a exatidão das estimativas obtidas com o Criterion, em relação à escalada. Já para analisar a precisão, foram observados os valores dos coeficientes de determinação $\left(\mathrm{R}^{2}\right)$ da regressão.

Além disso, para facilitar a comparação entre as variáveis e as diferentes distâncias, as variáveis estimadas foram analisadas utilizando as estatísticas complementares (Tabela 1) como o viés (V), média das diferenças absolutas (MD) e desvio padrão das diferenças (DPD) (FIGUEIREDO FILHO et al., 1996; CURTO et al., 2013), bem como os resíduos, todas relativizadas (\%). Para isso, o resultado de cada estatística foi dividido pela respectiva média da variável observada na escalada.

Tabela 1. Estatísticas complementares para avaliação do Criterion $\mathrm{RD} 1000^{\circledR}$ na estimativa do diâmetro, volume comercial e volume por segmento do fuste.

Table 1. Complementary statistics for the evaluation of Criterion RD $1000^{\circledR}$ in the estimation of diameter, commercial volume and volume by segment.

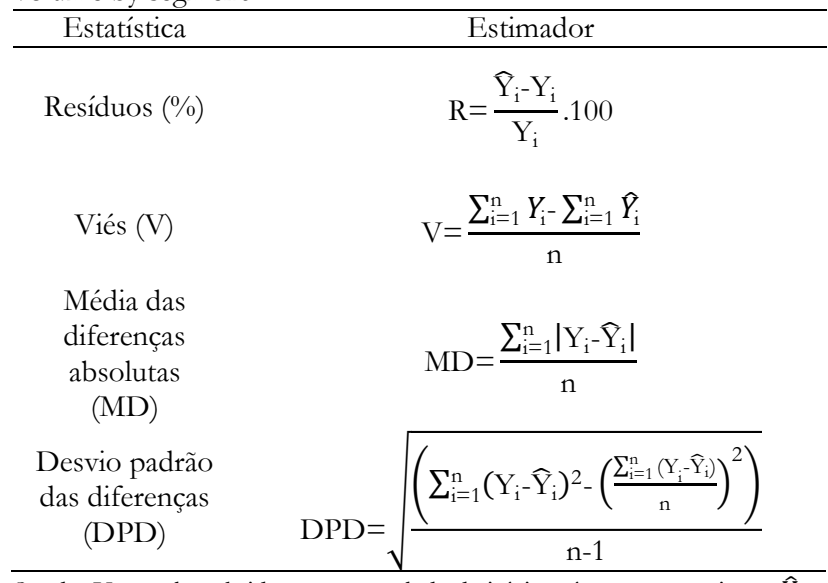

Sendo: $\mathrm{Y}_{\mathrm{i}}=$ valor obtido com a escalada da i-ésima árvore ou posição; $\widehat{Y}_{i}=$ valor estimado com o Criterion $\mathrm{RD} 1000^{\circledR}$ da i-ésima árvore ou posição medida; $\mathrm{n}=$ número de observações.

De posse dos resultados das estatísticas complementares, foi estruturada uma classificação com todas as distâncias mensuradas para as variáveis diâmetro, volume comercial e volume no segmento superior e inferior do fuste. Para cada estatística e variável, foram atribuídas notas de 1 a 4 , sendo 1 para a estatística com maior exatidão e 4 para a menor exatidão. Com o somatório das notas, foi possível classificar o desempenho do Criterion nas variações de distância de mensuração, sendo o melhor desempenho correspondente ao menor somatório (SILVA et al., 2012).

\section{RESULTADOS}

A altura comercial média das árvores foi de 13,46 m (desvio padrão de 2,87 m) e a distância média, com o máximo afastamento do equipamento em relação à árvore, foi de $19,39 \mathrm{~m}$ (desvio padrão de 2,41 m). O diâmetro médio ao longo do fuste foi de 48,33 cm (desvio padrão de 11,60 cm), e o volume comercial médio por árvore foi de $2,38 \mathrm{~m}^{3}$ (desvio padrão de $\left.0,98 \mathrm{~m}^{3}\right)$. As médias registradas e o resultado do teste $t$, para as distâncias e variáveis analisadas estão apresentados na Tabela 2.

Tabela 2. Média (p-valor) do diâmetro (di), volume comercial (vc), volume do segmento inferior (vi) e superior (vs) do fuste estimado com o Criterion RD $1000^{\circledR}$ em diferentes distâncias de mensuração. Table 2. Average (p-value) diameter (di), commercial volume (vc), volume of the lower (vi) and upper (vs) segment of the stem, of estimated value with the Criterion RD $1000^{\circledR}$ at different measurement distances.

\begin{tabular}{ccccc}
\hline Distâncias $(\mathrm{m})$ & $\mathrm{di}(\mathrm{cm})$ & $\mathrm{vc}\left(\mathrm{m}^{3}\right)$ & $\mathrm{vi}\left(\mathrm{m}^{3}\right)$ & $\mathrm{vs}\left(\mathrm{m}^{3}\right)$ \\
\hline \multirow{2}{*}{11} & 47,893 & 2,306 & 1,481 & 0,917 \\
& $(0,05)$ & $(0,09)$ & $(0,25)$ & $(0,09)$ \\
\hline \multirow{2}{*}{13} & 47,947 & 2,346 & 1,493 & 0,948 \\
& $(0,10)$ & $(0,49)$ & $(0,63)$ & $(0,46)$ \\
\hline \multirow{2}{*}{15} & 47,942 & 2,345 & 1,500 & 0,940 \\
& $(0,07)$ & $(0,41)$ & $(0,84)$ & $(0,26)$ \\
\hline \multirow{2}{*}{$>$ Dist. } & 47,898 & 2,342 & 1,500 & 0,936 \\
& $(0,05)$ & $(0,36)$ & $(0,85)$ & $(0,18)$ \\
\hline
\end{tabular}

Em que: $p$-valor $>0,05$ indica diferença não significativa; $>$ Dist $=$ maior distância possível entre a árvore e o equipamento.

O teste de Bartlett indicou homogeneidade das variâncias para o diâmetro ao longo do fuste $(p$-valor $=0,9994)$, para o volume comercial $(p$-valor $=0,9980)$ e para o volume no segmento superior e inferior $(p$-valor $=0,9897)$. Já o teste $t$ demonstrou não existir diferença significativa entre as médias dos valores reais obtidos na cubagem por escalada e os estimados pelo equipamento Criterion, para qualquer distância avaliada (Tabela 2).

Analisando os resíduos para a estimativa dos diâmetros ao longo do fuste (Figura 1), pode-se verificar que estes possuem uma amplitude inferior a $\pm 25 \%$, com tendência à subestimativa do diâmetro nas partes mais altas do fuste, para todas as distâncias, sendo isso menos evidente na maior distância adotada. Este comportamento se acentua ao longo do fuste, onde os erros aumentam na medida que os diâmetros diminuem.

Observa-se uma relação linear entre os diâmetros observados na escalada e os estimados com o Criterion (Figura 2). O coeficiente $\beta_{1}$ apresentou valores próximos de 1 (um), indicando a inclinação da reta próxima de $45^{\circ} \mathrm{e}$ moderada exatidão nas estimativas. Além disso, os valores de coeficiente de determinação $\left(\mathrm{R}^{2}\right)$ foram superiores a 0,9 , retratando que existe precisão na estimativa.

No geral, verifica-se que para as árvores de maiores volumes comerciais houve tendência em subestimativa em todas as distâncias de posicionamento do aparelho, já nos menores volumes comerciais ocorreu o inverso, mesmo que com baixa magnitude (Figura 3).

Ao analisar a tendência do volume comercial por segmento do fuste estimados em função dos valores observados (Figura 4), não foram verificadas diferenças evidentes no segmento inferior do fuste, ou seja, de 2,0 a 8,0 $\mathrm{m}$ de altura, nas diferentes distâncias avaliadas. Porém, maiores erros de estimativa foram observados no segmento superior do fuste, com tendência a subestimativas, principalmente quando o aparelho é posicionado mais próximo da árvore (Figura 4). Isso é comprovado a partir dos valores de $\beta_{1}$ serem inferiores a 1 (um) e a maior dispersão dos pontos em torno da reta, evidenciado pelos menores 
valores de coeficiente de determinação $\left(\mathrm{R}^{2}\right)$ (Figura 4).

Já ao considerar as análises estatísticas complementares de viés $(\mathrm{V})$, média das diferenças absolutas (MD) e desvio padrão das diferenças (DPD) observam-se variações não evidenciadas com as análises anteriores ao analisar as diferentes distâncias entre o observador e a árvore (Tabela 3). Valores de viés próximos de 0 (zero) indicam que não há tendência nas estimativas dos diâmetros, volume comercial e volume no segmento inferior. Apenas verifica-se tendência de subestimativa quando se avalia o volume do segmento superior do fuste, apresentando valores de viés de até 5,63\% na menor distância $(11 \mathrm{~m})$.

Para o volume do segmento superior do fuste, verificouse maiores amplitudes dos resíduos, ao analisar a média das diferenças absolutas (MD), bem como maiores valores para desvio padrão das diferenças (DPD), indicando menor homogeneidade dos resíduos em comparação com o segmento inferior do fuste.

Os valores mais elevados de V, MD e DPD foram observados para as menores distâncias do equipamento, indicando que, quanto menor a distância, maiores foram as tendências em subestimar as variáveis $(\mathrm{V})$, maiores as amplitudes de erro (MD) e menos homogêneos foram os resíduos (DPD).

De modo geral, houve aumento dos erros quando se optou pela menor distância da árvore, entretanto, os menores erros não foram observados na maior distância possível em relação às árvores. $\mathrm{O}$ melhor desempenho do Criterion foi observado a uma distância de $15 \mathrm{~m}$ entre a árvore e o equipamento. Entretanto, para o volume do segmento superior do fuste, o melhor desempenho foi conseguido à máxima distância possível da árvore.
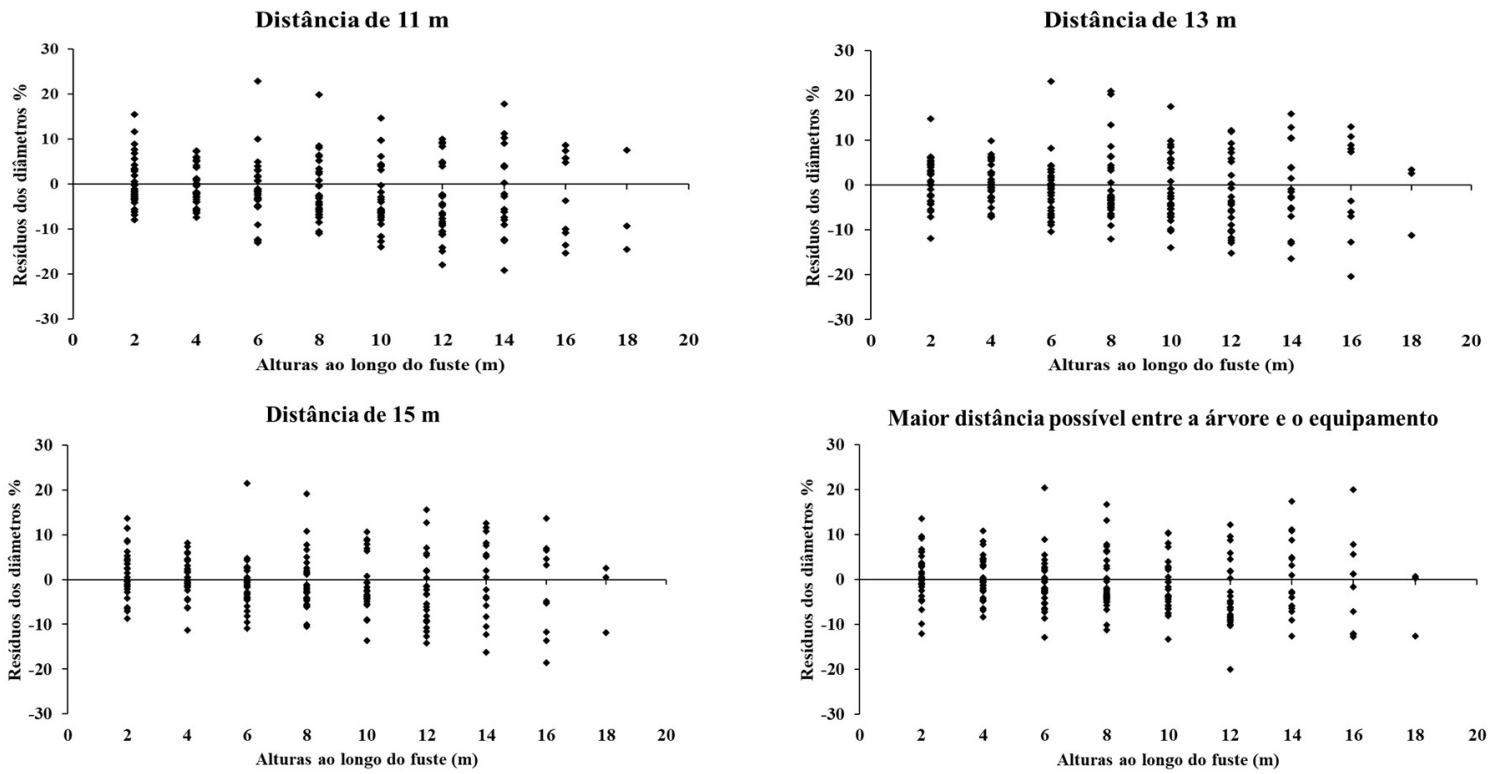

Figura 1. Dispersão dos resíduos para os diâmetros ao longo do fuste estimados com o Criterion RD $1000^{\circledR}$ em diferentes distâncias de mensuração.

Figure 1. Dispersion of the residues for the diameters estimated with Criterion RD 1000® as a function of heights along the stem.
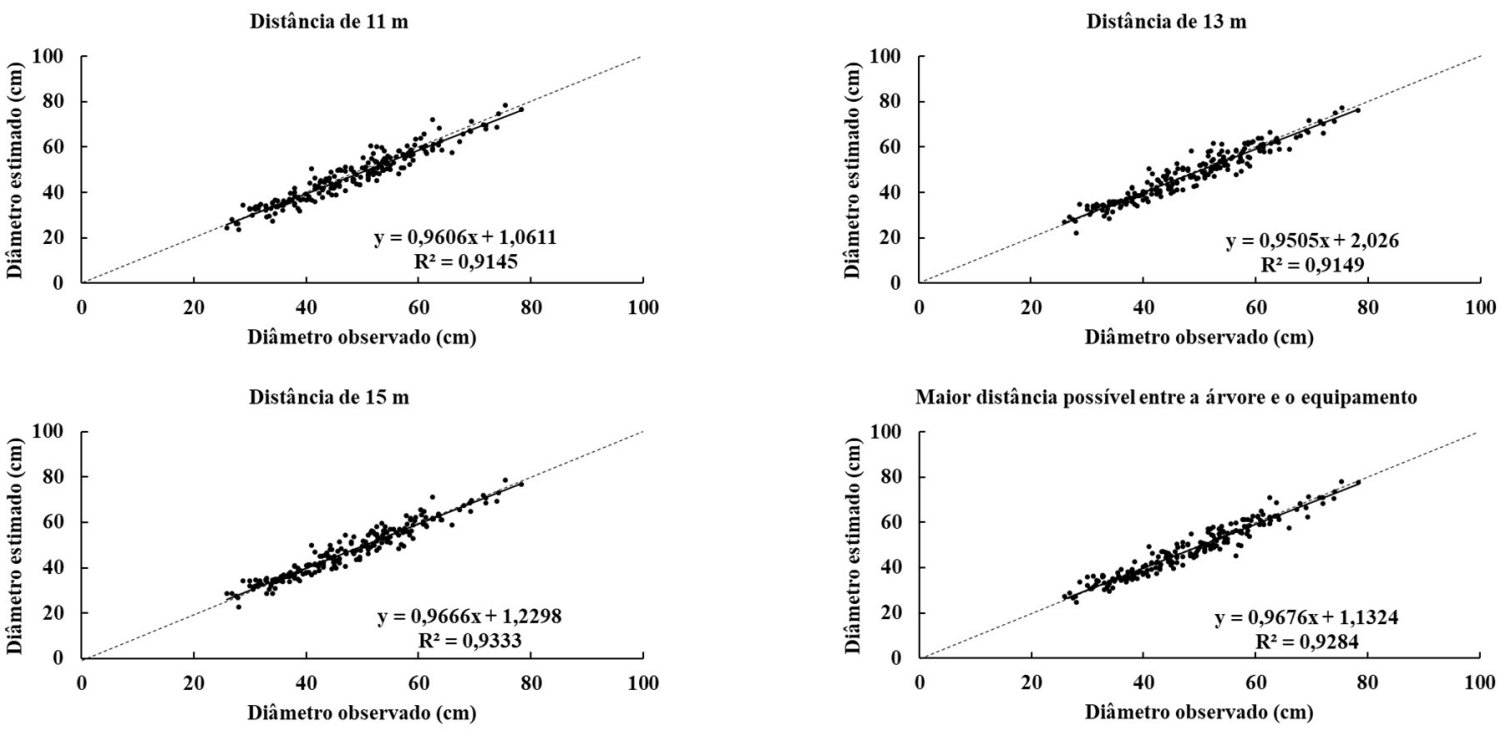

Figura 2. Relação entre diâmetros observados obtidos com a escalada das árvores e estimados com o Criterion RD $1000^{\circledR}$, em diferentes distâncias de mensuração. (Nota: Linhas tracejadas indicam inclinação de $45^{\circ}$ ).

Figure 2. Relationship between diameters observed obtained with tree climbing and estimated with Criterion RD $1000^{\circledR}$, at different measurement distances. (Nota: Linhas tracejadas indicam inclinação de $45^{\circ}$ ). 


\section{Curto et al.}
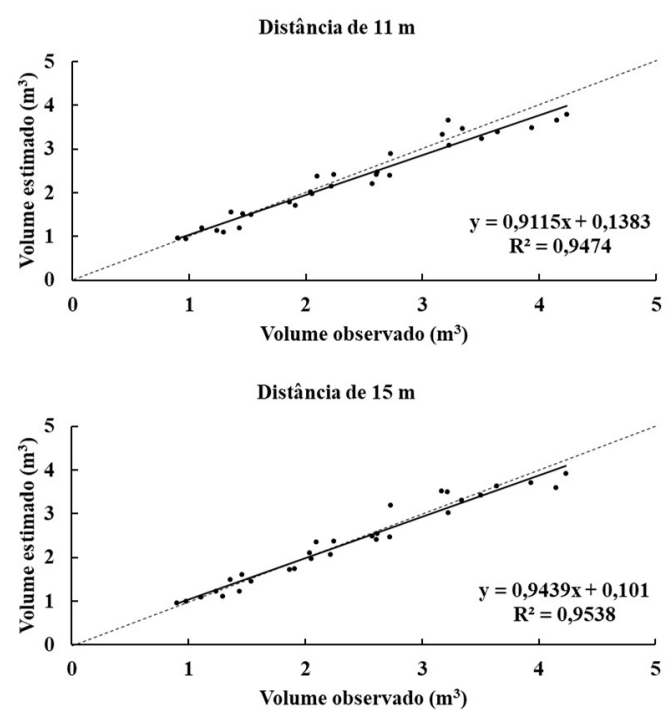

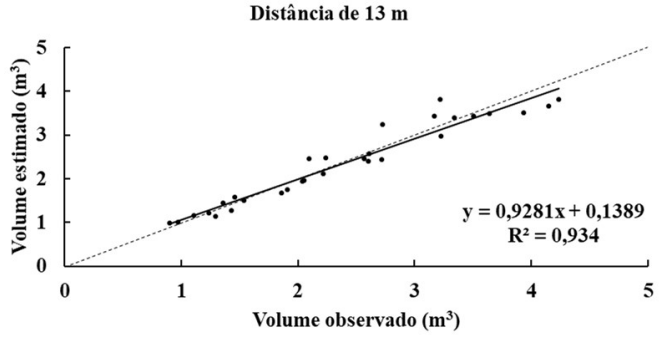

Maior distância possível entre a árvore e o equipamento

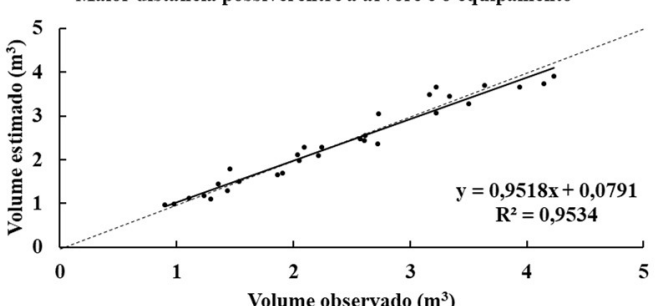

Figura 3. Relação entre o volume comercial observado e o volume comercial estimado, em diferentes distâncias de mensuração.

Figure 3. Relationship between the observed commercial volume and the estimated commercial volume, at different measurement distances. Nota: Linhas tracejadas indicam inclinação de $45^{\circ}$

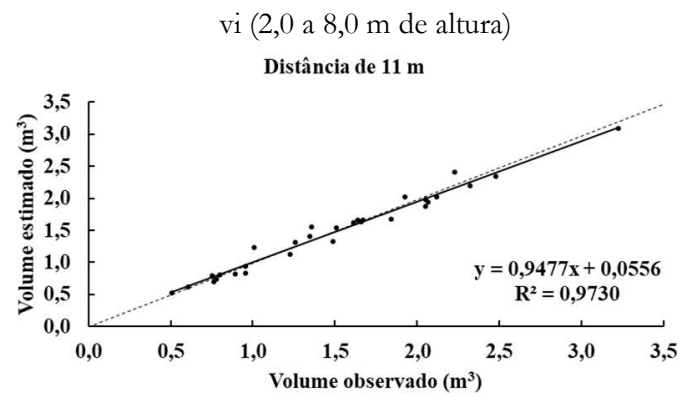

Distância de $13 \mathrm{~m}$
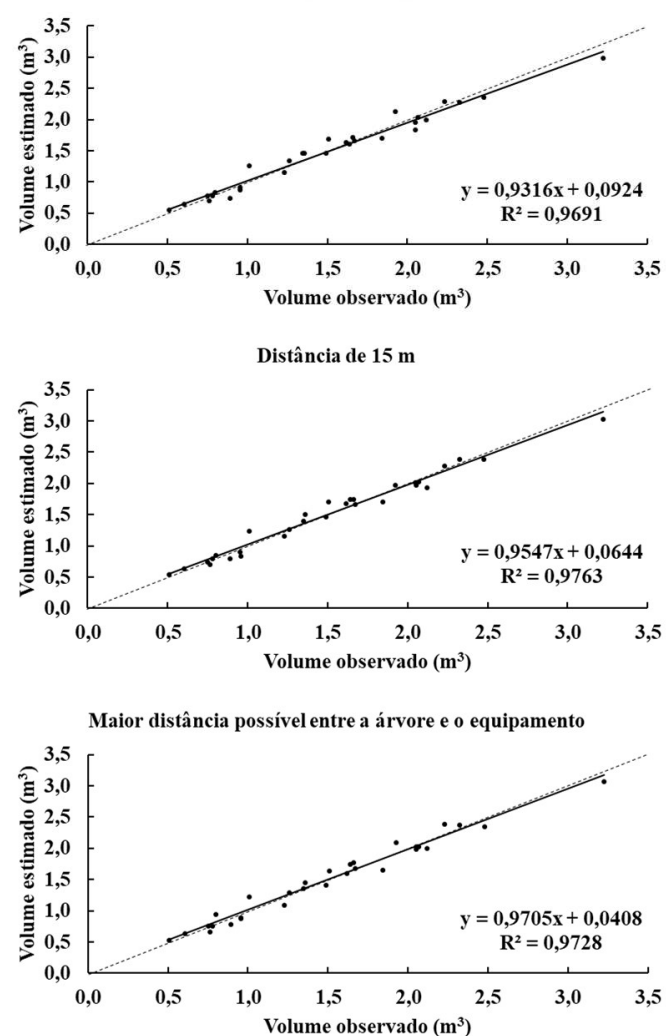

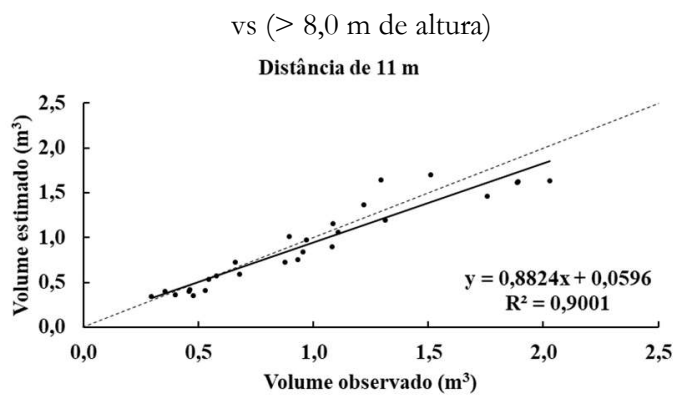

Distância de $13 \mathrm{~m}$

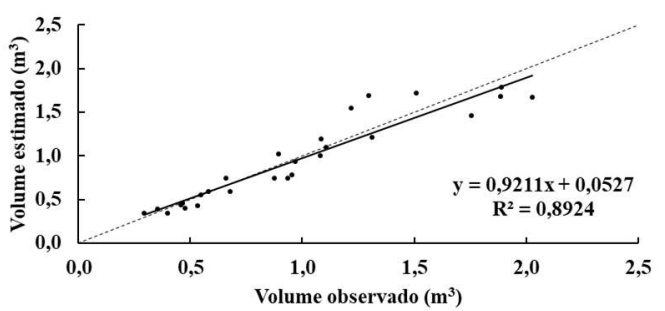

Distância de 15 m

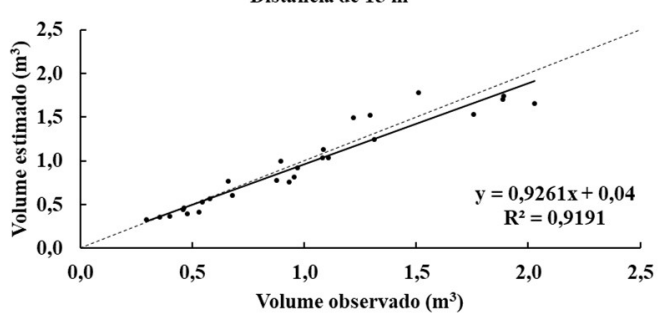

Maior distância possível entre a árvore e o equipamento

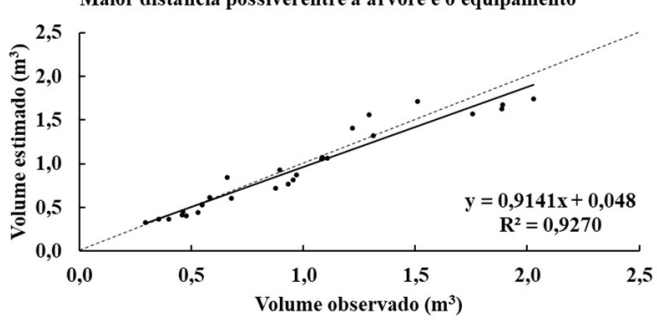

Figura 4. Relação entre o volume observado e o volume estimado nos segmentos inferior (vi) e superior (vs) do fuste, em diferentes distâncias de mensuração. 
Figure 4. Relationship between the observed and estimated volume in the lower (vi) and upper (vs) segments of the stem, at different measurement distances.

Nota: Linhas tracejadas indicam inclinação de $45^{\circ}$.

Tabela 3. Estatísticas complementares e notas atribuídas para as estimativas de diâmetro (di), volume comercial (vc) e volume do segmento inferior (vi) e superior (vs) do fuste, em diferentes distâncias de mensuração.

Table 3. Complementary statistics and grade attributed to the statistics evaluating diameter, commercial volume, and volume in the lower (vi) and upper (vs) segments of the stem, at different measurement distances.

\begin{tabular}{|c|c|c|c|c|c|c|c|c|}
\hline Variável & Distâncias (m) & $\mathrm{V}(\%)$ & Nota & $\mathrm{MD}(\%)$ & Nota & DPD $(\%)$ & Nota & $\sum$ Notas \\
\hline \multirow{4}{*}{ di } & 11 & 1,74 & 4 & 5,78 & 4 & 7,10 & 4 & 12 \\
\hline & 13 & 0,79 & 1 & 5,54 & 3 & 6,91 & 3 & 7 \\
\hline & 15 & 0,80 & 2 & 4,98 & 1 & 6,32 & 1 & 4 \\
\hline & > Dist. & 0,90 & 3 & 5,04 & 2 & 6,35 & 2 & 7 \\
\hline \multirow{4}{*}{$\mathrm{vc}$} & 11 & 3,04 & 4 & 8,13 & 3 & 9,93 & 3 & 10 \\
\hline & 13 & 1,35 & 1 & 8,18 & 4 & 10,55 & 4 & 9 \\
\hline & 15 & 1,36 & 2 & 6,89 & 1 & 8,87 & 1 & 4 \\
\hline & $>$ Dist. & 1,50 & 3 & 7,24 & 2 & 8,92 & 2 & 7 \\
\hline \multirow{4}{*}{$\begin{array}{c}\mathrm{vi} \\
(2,0 \text { a } 8,0 \mathrm{~m})\end{array}$} & 11 & 1,53 & 4 & 5,76 & 3 & 7,24 & 3 & 10 \\
\hline & 13 & 0,69 & 3 & 6,02 & 4 & 7,68 & 4 & 11 \\
\hline & 15 & 0,25 & 2 & 5,26 & 1 & 6,61 & 1 & 4 \\
\hline & $>$ Dist. & 0,24 & 1 & 5,74 & 2 & 7,01 & 2 & 5 \\
\hline \multirow{4}{*}{$\begin{array}{c}\text { vs } \\
(>8,0 \mathrm{~m} \text { de altura })\end{array}$} & 11 & 5,63 & 4 & 13,52 & 4 & 17,28 & 4 & 12 \\
\hline & 13 & 2,48 & 1 & 12,87 & 3 & 17,17 & 3 & 7 \\
\hline & 15 & 3,28 & 2 & 11,36 & 2 & 15,04 & 2 & 6 \\
\hline & > Dist. & 3,65 & 3 & 11,02 & 1 & 14,43 & 1 & 5 \\
\hline
\end{tabular}

Legenda: $\mathrm{V}=$ viés; $\mathrm{MD}=$ média das diferenças absolutas; DPD = desvio padrão das diferenças; $>$ Dist $=$ maior distância possível entre a árvore e o equipamento; $\sum$ somatório.

\section{DISCUSSÃO}

Os valores médios de diâmetro e volumes estimados pelo Criterion não apresentam diferenças estatisticamente significativas do valor observado, obtido diretamente com a escalada da árvore. Estes resultados se assemelham aos obtidos em plantio de Cryptomeria japonica (Thunb. Ex L. f.) D. Don utilizando o mesmo dendrômetro digital, os quais não encontraram diferenças estatisticamente significativas para diâmetro e volume (CORTE et al., 2016). O mesmo comportamento foi observado ao comparar as médias da cubagem destrutiva com as obtidas pelo Criterion, em plantios de Pinus sp. na Cordilheira Ibérica do Norte da Espanha (RODRIGUEZ et al., 2014), em plantio de Khaya ivorensis em Minas Gerais (OLIVEIRA et al., 2018) e em sistemas integração lavoura pecuária-floresta, com o clone Eucalyptus grandis x Eucalyptus urophylla, no Mato Grosso (CURTO et al., 2019). Assim, as características destes ambientes não apresentaram influência na média estimada das variáveis, de modo que o uso do equipamento é recomendado em tais condições.

Para as estimativas dos diâmetros ao longo do fuste (Figura 1), em qualquer uma das distâncias avaliadas, foram verificadas tendências de subestimativa dos menores diâmetros. Nicoletti et al. (2015b) ao avaliarem a exatidão do Criterion RD $1000^{\circledR}$ em um remanescente florestal de Mata Atlântica, também observaram tendência de subestimativa dos diâmetros e afirmaram que estes erros podem estar relacionados à dificuldade de visualização dos diâmetros menores ao longo do fuste, uma vez que em árvores de menores diâmetros notaram maiores erros em relação às de maior porte.

Dificuldades ao utilizar o Criterion RD $1000^{\circledR}$ para mensuração dos menores diâmetros localizados nas maiores alturas ocorreram também devido a barra laser que mede o diâmetro no visor do equipamento ser maior que o diâmetro a ser mensurado, ampliando assim os erros, conforme verificado em plantio de Pinus taeda com árvores de até $28 \mathrm{~m}$ de altura e em um remanescente florestal com indivíduos de diâmetros diminutos (BONAZZA et al., 2015; NICOLETTI et al., 2015b).

Rodriguez et al. (2014) analisaram o aparelho Criterion RD $1000^{\circledR}$ e verificaram maiores variações de amplitudes dos erros para diâmetros estimados em alturas superiores do fuste. Estes resultados foram semelhantes aos do presente estudo, onde foram observadas maiores amplitudes nos erros para o segmento acima de $8 \mathrm{~m}$ de altura em relação aos erros para o segmento abaixo de $8 \mathrm{~m}$ de altura (Figura 4 e Tabela 3). Porém, no presente estudo, por se tratarem de árvores com maiores diâmetros as que analisadas por Rodriguez et al. (2014), Bonazza et al. (2015), Nicoletti et al. (2015b) e Corte et al. (2016), pode-se afirmar que esta amplitude do erro está relacionado ao posicionamento inclinado do equipamento para realizar as medições.

As estimativas de volume comercial também apresentaram maiores erros considerando os menores diâmetros, localizados no segmento superior do fuste (Figura 4) e visivelmente com maior amplitude dos erros em relação aos maiores diâmetros, localizados na base do fuste (Figura 4). Em concordância a esses resultados, Bonazza et al. (2015) em plantio de Pinus taeda e Corte et al. (2016) em plantio de Cryptomeria japonica concluíram que as estimativas volumétricas obtidas a partir das variáveis mensuradas pelo Criterion $\mathrm{RD} 1000^{\circledR}$ são mais exatas para árvores de maiores dimensões, com maiores amplitudes de erros nas porções superiores do fuste.

Em relação a distância do mensurador a árvore, ocorreu o aumento de erros quando se optou pela menor distância da árvore. Isso ocorre porque, o operador ao ficar mais próximo à árvore, faz com que o aparelho seja manuseado de forma mais inclinada. Devido a isso, qualquer oscilação no momento da medição pode resultar em erros grosseiros, quando do uso de aparelhos baseados no princípio 
trigonométrico, conforme avaliado em florestas nativas por Silva et al. (2012). Outro fator que pode justificar o aumento dos erros no segmento superior do fuste é a posição desconfortável do operador na realização das medições das maiores alturas (BONAZZA et al., 2015), devido à necessidade de maior inclinação do pescoço.

Ainda com relação a distância do mensurador a árvore, os melhores resultados foram obtidos quando o Criterion esteve a $15 \mathrm{~m}$ de distância, sendo uma distância $10 \%$ superior à média da altura comercial das árvores $(13,46 \mathrm{~m} \pm 2,87 \mathrm{~m})$. A adoção de distância próxima à altura média das árvores apresentou um melhor desempenho para a mensuração da árvore com o Criterion conforme Rodriguez et al. (2014) e Bonazza et al. (2015).

Quando a distância do operador a árvore foi muito grande (em média 19,39 $\mathrm{m} \pm 2,41 \mathrm{~m}$ ) a exatidão tendeu a diminuir (Tabela 3). Estes resultados também podem estar associados à densidade do sub-bosque da floresta, representando pequenos obstáculos à frente da lente de observação do equipamento (RODRIGUEZ et al., 2014), sendo que quanto maior a distância, maior a presença desses obstáculos. Porém, observa-se melhor desempenho ao utilizar a maior distância adotada (média de 19,39 m) na estimativa do volume do segmento superior do fuste. Tal fato pode ser explicado pela menor inclinação do equipamento, visto que quando o equipamento está muito próximo da árvore a ser medida, obriga o mensurador a incliná-lo, de modo a ocasionar erros se qualquer oscilação existir no momento da leitura.

Algumas outras dificuldades ocorrem nas medições em maiores distâncias utilizando relascópio a laser, com distorções na medição, quando há presença de sub-bosque adensado, com presença de galhos, líquens e cascas parcialmente soltas no fuste (KALLIOVIRTA et al., 2005), bem como problemas com a iluminação no interior da floresta (WILLIAMS et al., 1999). Estas dificuldades são semelhantes na região do estudo, onde a floresta Estacional Semidecidual de dossel emergente do bioma amazônico, apresenta diversos impedimentos para a correta medição dos diâmetros com o equipamento, como arbustos, árvores jovens, cipós e pela característica da espécie em estudo em desprender sua casca abundantemente.

\section{CONCLUSÕES}

O Criterion RD $1000^{\circledR}$ apresentou exatidão na estimativa de diâmetro e volume de árvores em pé, viabilizando a cubagem não destrutiva de Qualea sp. em florestas do bioma Amazônia.

A distância de $15 \mathrm{~m}$, correspondendo a distância de até $10 \%$ superior à média da altura comercial das árvores, apresentou o melhor desempenho do equipamento na estimativa do diâmetro e volume comercial.

\section{AGRADECIMENTOS}

Os autores agradecem à empresa Floresta Assessoria e Planejamento Florestal Ltda e à Embrapa Agrossilvipastoril, localizadas em Sinop, Mato Grosso, e a Fundação de Amparo à Pesquisa do Estado de Mato Grosso/FAPEMAT por conceder bolsa de estudos a um dos autores.

\section{REFERÊNCIAS}

ALVARES, C. A.; STAPE, J. L.; SENTELHAS, P. C.; DE
MORAES GONÇALVES, J. L.; SPAROVEK, G. Köppen's climate classification map for Brazil. Meteorologische Zeitschrift, v. 22, n. 6, p. 711-728, 2013. DOI: http://dx.doi.org/10.1127/09412948/2013/0507.

ARAÚJO, R. A.; COSTA, R. B.; FELFILI, J. M.; KUNTZ, I. G.; SOUZA, R. A. T. de M. e; DORVAL, A. Florística e estrutura de fragmento florestal em área de transição na Amazônia Mato-grossense no município de Sinop. Acta Amazônica, Manaus, v. 39, n. 4, p. 865-877, 2009. DOI: https://doi.org/10.1590/S0044-59672009000400015.

BONAZZA, M.; SAMPIETRO, J. A.; SILVESTRE, R.; NICOLETTI, M. F.; LIMA, G. C. P.; SILVA, A. L. da.; MORÉS, D. F.; RODRIGUES, A. L. Accuracy of nondestructive volumetric estimates in stands of Pinus taeda L. Australian Journal of Basic and Applied Sciences, v. 9, n. 31, p. 71-78, 2015.

BRASIL. Ministério do Meio Ambiente. Instrução Normativa $\mathrm{n}^{\circ}$ 5, de 11 de dezembro de 2006. Dispõe sobre procedimentos técnicos para elaboração, apresentação, execução e avaliação técnica de Planos de Manejo Florestal Sustentável-PMFSs nas florestas primitivas e suas formas de sucessão na Amazônia Legal, e dá outras providências. Diário Oficial da República Federativa do Brasil, Brasília, DF, 2006.

BRASIL. Resolução CONAMA n ${ }^{\circ}$ 406/2009. Estabelece parâmetros técnicos a serem adotados na elaboração, apresentação, avaliação técnica e execução de Plano de Manejo Florestal Sustentável-PMFS com fins madeireiros, para florestas nativas e suas formas de sucessão no bioma Amazônia. Diário Oficial da República Federativa do Brasil, Brasília, no 26, p. 100, de 06 de fev. 2009. Seção 1.

CORTE, A. P. D.; SANQUETTA, C. R.; OLIVEIRA, K. A.; BEHLING, A.; COUTINHO, V. M. Desempenho de diferentes equipamentos para mensuração de diâmetro a $1,30 \mathrm{~m}$, altura individual total e volume do fuste $\mathrm{em}$ Cryptomeria japonica (Thunb. Ex L. f.) D. Don. Enciclopédia Biosfera, Goiânia, v. 13, n. 23, p. 432-441, 2016.

DOI: http://dx.doi.org/10.18677/Enciclopedia_Biosfera_201 6_038.

CURTO, R. A.; LAURO, A. C.; TONINI, H.; KOHLER, S. V.; ARAÚJO, E. J. G.; BIAZATTI, S. C. Cubagem de árvores em pé com dendrômetro óptico em sistema de integração lavoura-pecuária-floresta. Pesquisa florestal Brasileira, Colombo, v. 39, p. 1-11, 2019. DOI: https://doi.org/10.4336/2019.pfb.39e201801646

CURTO, R. A.; SILVA, G. F.; SOARES, C. P. B.; MARTINS, L. T.; DAVID, H. C. Métodos de estimação de altura de árvores em Floresta Estacional Semidecidual, Floresta, Curitiba, v. 43, n. 1, p. 105-116, 2013. DOI: http://dx.doi.org/10.5380/rf.v43i1.26791

FIGUEIREDO FILHO, A; BORDERS, B. E.; HITCH K. L. Taper equations for Pinus taeda plantations in Southern Brazil. Forest Ecology and Management, Amsterdam, v. 83, p. 39-46, 1996. DOI: https://doi.org /10.1016/0378-1127(96)03706-1

GAMA, J. R. V.; BOTELHO, S. A.; BENTES-GAMA, M. M.; SCOLFORO, J. R. S. Estrutura e potencial futuro de utilização da regeneração natural de floresta de várzea alta no município de Afuá, estado do Pará. Ciência Florestal, Santa Maria, v. 13, n. 2, p. 71-82, 2003. DOI: 
https://doi.org/10.5902/198050981744.

KALLIOVIRTA, J.; LAASASENAHO, J; KANGAS, A. Evaluation of the Laser-relascope. Forest Ecology and Management, Amsterdam, v. 204, p. 181-194, 2005. DOI: $10.1016 /$ j.foreco.2004.09.020

MOTA L. L.; BOTON D.; FONSECA, R. C.; SILVA W. C.; SOUZA A. P. Balanço hídrico climatológico e classificação climática da região de Sinop, Mato Grosso. Scientific Electronic Archives, Rondonópolis, v. 3, p. 38-44, 2013.

DOI: http://dx.doi.org/10.36560/30201339

NICOLETTI, M. F.; BATISTA, J. L. F.; CARVALHO, S. P. C.; CASTRO, T. N.; HESS, A. F. Exatidão de dendrômetros ópticos para determinação do volume de árvores em pé. Ciência Florestal, Santa Maria, v. 25, n. 2, p. 395-404, 2015a. DOI: https://doi.org/10.5902/1980509818458

NICOLETTI, M. F.; SILVA, E; FLORIANI, M. M. P. Metodologia não destrutiva para quantificação do volume e biomassa do fuste em remanescente florestal. Nativa, Sinop, v. 3, n. 4, p. 287-291, 2015b. DOI: http://dx.doi.org/10.14583/23187670.v03n04a11

NICOLETTI, M. F.; CARVALHO, S. P. C.; BATISTA, J. L. F. Revisão bibliográfica sobre métodos não-destrutivos de cubagem de árvores em pé visando à determinação da biomassa. Revista Científica Eletrônica de Engenharia Florestal, Garça, v. 20, n. 1, p. 102-116, 2012.

RODRIGUEZ, F.; LIZARRALDE, I.; FERNÁNDEZLANDA, A.; CONDÉS, S. Non-destructive measurement techniques for taper equation development: a study case in the Spanish Northern Iberian Range. European Journal of Forest Research, Freising, v. 133, p. 213-223, 2014. DOI: https://doi.org/10.1007/s10342-013-0739-5

SANQUETTAA, C. R.; PIVA, L. R.; WOJCIECHOWSKI, J.; CORTE, A. P. D.; SCHIKOWSKI, A. B. Volume estimation of Cryptomeria japonica logs in southern Brazil using artificial intelligence models. Southern Forests, v. 1, p. $1-8, \quad 2017 . \quad$ DOI: https://doi.org/10.2989/20702620.2016.1263013

SILVA, G. F.; MÔRA, R.; CURTO, R. de A. Simulação de erros na medição de altura de árvores inclinadas com aparelhos baseados em princípios trigonométricos. Nativa, Sinop, v. 5. n. 5, p. 372-379. 2017. DOI: http://dx.doi.org/10.5935/2318-7670.v05n05a12 SILVA, G. F.; CURTO, R. A.; SOARES, C. P. B.; PIASSI, L. C. Avaliação de métodos de medição de altura em florestas naturais. Revista Árvore, Viçosa, v. 36, n. 2, p. 341-348, 2012. DOI: https://doi.org/10.1590/S010067622012000200015

SOUZA, H. S.; DRESCHER, R.; VENDRUSCOLO, D. G. S.; MOURA, J. P. V. M.; SIQUEIRA, T. A. S.; MAMORÉ, F. M. D. Comparação de métodos de cubagem para eucalipto. Revista Brasileira de Biometria, Lavras, v. 35, n. 1, p. 17-26, 2017.

WILlIAMS, M. S.; CORNIER, K.; BRIGGS, R.; MARTINEZ, D. Evaluation of the Barr \& Stroud FP15 and Criterion 400 laser dendrometers for measuring upper stem diameters and heights. Forest Science, Oxford, v.45, n.1, p. 53-61. 1999. DOI: https://doi.org/10.1093/forestscience/4 